\title{
Iperparatiroidismo primitivo e nefrolitiasi: vi è spazio per $i$ calciomimetici?
}

\author{
M. Marangella, C. Vitale \\ S.C. Nefrologia e Dialisi, A.O. Ordine Mauriziano Umberto I, Torino
}

\section{Epidemiologia}

Liperparatiroidismo primitivo (IPTP) è la disendocrinopatia più frequente dopo il diabete mellito e le tireopatie. Nella popolazione generale, è la principale causa di ipercalcemia asintomatica. Nel 70-80\% dei casi di IPTP, la causa è un adenoma paratiroideo isolato, mentre un'iperplasia diffusa di tutte le ghiandole è riscontrabile nel $15-20 \%$ dei casi. L'incidenza degli adenomi paratiroidei è 2-3 volte superiore nelle femmine rispetto ai maschi. É tuttavia probabile che la prevalenza di IPTP sia sottostimata nella popolazione generale, poiché in molti casi si tratta di patologia clinica- mente asintomatica.

In anni recenti, è stata definita una condizione di Iperparatiroidismo normocalcemico, nella quale agli elevati livelli di PTH corrispondono calcemie normali o solo borderline (1).

Fra i disturbi più frequentemente associati a IPTP è da ricordare la nefrolitiasi calcica, che ricorre in circa il $60 \%$ dei pazienti. A volte la diagnosi di IPTP viene fatta su pazienti in cui una ecografia occasionale ha rivelato una calcolosi misconosciuta.

\section{Trattamento medico}

La maggior parte degli esperti della materia ritiene che la terapia definitiva di IPTP sia la paratiroidectomia (PTX). Questo nonostante che molti dei pazienti non operati, a partire dal momento della diagnosi, non dimostrino un andamento evolutivo della patologia. Pazienti con IPTP seguiti per un follow-up fino a 10 e 15 anni non hanno mostrato un peggioramento del profilo biochimico di calcemia e $\mathrm{PTH}$, né peggioramento di funzione renale o di ipertensione arteriosa $(2,3)$. Tuttavia, l'incidenza di effetti collaterali o complicanze è significativamente maggiore nei pazienti non operati rispetto a quelli sottoposti a PTX. Infatti l'incidenza di calcolosi e delle sue recidive e l'andamento densitometrico dell'osso, soprattutto di quello spugnoso, sono peggiori nei non operati (4).

Sulla base di queste osservazioni un panel di esperti negli USA ha ancora di recente confermato l'opportunità di proporre la PTX ai pazienti con complicanze ossee o renali di $\operatorname{IPTP}(5,6)$. Fra i criteri aggiuntivi che consigliano la PTX vi sono:

1. calcemia di $1 \mathrm{mg} / \mathrm{dL}$ al di sopra del limite superiore di norma;

2. calciuria $>400 \mathrm{mg} / 24$ ore;

3. riduzione della funzione renale superiore al $30 \%$;

4. densitometria ossea con $\mathrm{T}$ score $<-2.5$; 5. età $<50$ anni.

È unanimemente ammesso che la chirurgia efficace è l'unica procedura nota in grado di correggere in via definitiva la disfunzione paratiroidea.

Fatte salve le considerazioni su esposte esiste ancora una quota di pazienti che devono essere gestiti con terapia medica, in particolare quelli:

1. non operabili per elevato rischio operatorio;

2. con patologie intercorrenti;

3. che rifiutano l'intervento;

4. che presentano recidiva di PHP comportante difficoltà nell'approccio operatorio;

5. in lista di attesa per intervento.

Le raccomandazioni di gestione medica dei pazienti sono discusse qui di seguito. 
Fig. 1 - Patogenesi di IPTP con massa nefronica conservata.

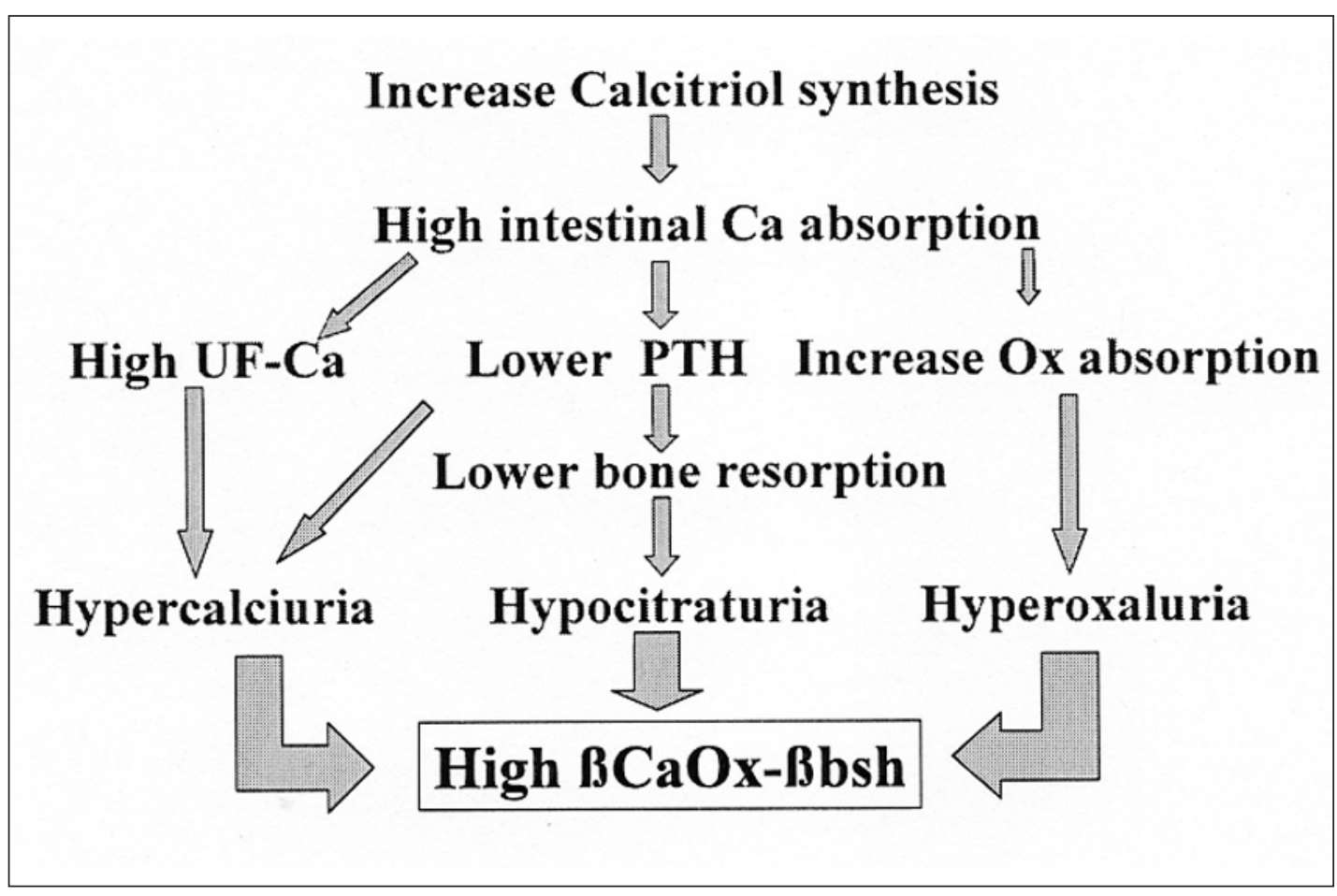

\section{Mantenimento in follow-up}

Va effettuato invitando il paziente alla esecuzione periodica di esami. Le indicazioni fatte dal panel di esperti sono schematizzate in Tabella I (5). L'esclusione della misura della clearance della creatinina è giustificata dalla possibilità di un calcolo indiretto mediante la formula di Cockcroft-Gault. Poiché la calcolosi renale è la manifestazione clinica più frequente nei pazienti sintomatici, l'esclusione di calciuria ed ecografia renovescicale desta qualche perplessità. Si tenga anche presente che le recidive di calcolosi sono più frequenti fra i non operati rispetto a quelli sottoposti a PTX (7). Calciuria ed Eco reno-vescicale devono essere incluse

TABELLA I - INDAGINI PERIODICHE NEL FOLLOW-UP DI PAZIENTI CON IPTP NON OPERATI*

\begin{tabular}{lc}
\hline Esame & Periodicità \\
\hline Calcemia & 6 mesi \\
Calciuria & Non raccomandata \\
Clearance creatininica & Non raccomandata \\
Creatinina sierica & Annuale \\
Osteodensitometria & Annuale \\
Eco ed Rx Diretta apparato urinario & Non raccomandata \\
\hline
\end{tabular}

*Workshop on Asymptomatic primary hyperparathyroidism, 2002. fra le indagini basali. Molto raccomandata è la periodica valutazione densitometrica dell'osso, da effettuare in tre segmenti: lombare, collo femorale, radio distale.

\section{Idratazione e prescrizioni dietetiche}

La frequenza della calcolosi in IPTP e la possibile poliuria da ipercalcemia, consigliano di mantenere un apporto di liquidi regolare ed adeguato. La dieta deve prevedere un apporto calcico analogo a quello raccomandato nella popolazione generale, e pertanto intorno a 0.8-1.0 g/die. Una eventuale moderata riduzione di calcio dietetico potrà essere prescritta ai pazienti che abbiano elevati livelli di calcitriolo plasmatico. Una dieta ipocalcica espone al rischio di peggioramento della osteopenia e non protegge dal rischio di calcolosi renale anche perché causa di iperossaluria da aumentato assorbimento intestinale di ossalato.

\section{Terapia farmacologica}

Vitamina $D$. In condizioni di normale funzione renale i pazienti con IPTP mostrano elevati livelli plasmatici di calcitriolo e pertanto non necessitano di terapia con vitamina D. Tuttavia, uno stato carenziale severo, valutabile con il dosaggio di $25(\mathrm{OH}) \mathrm{D} 3$ può accentuare 


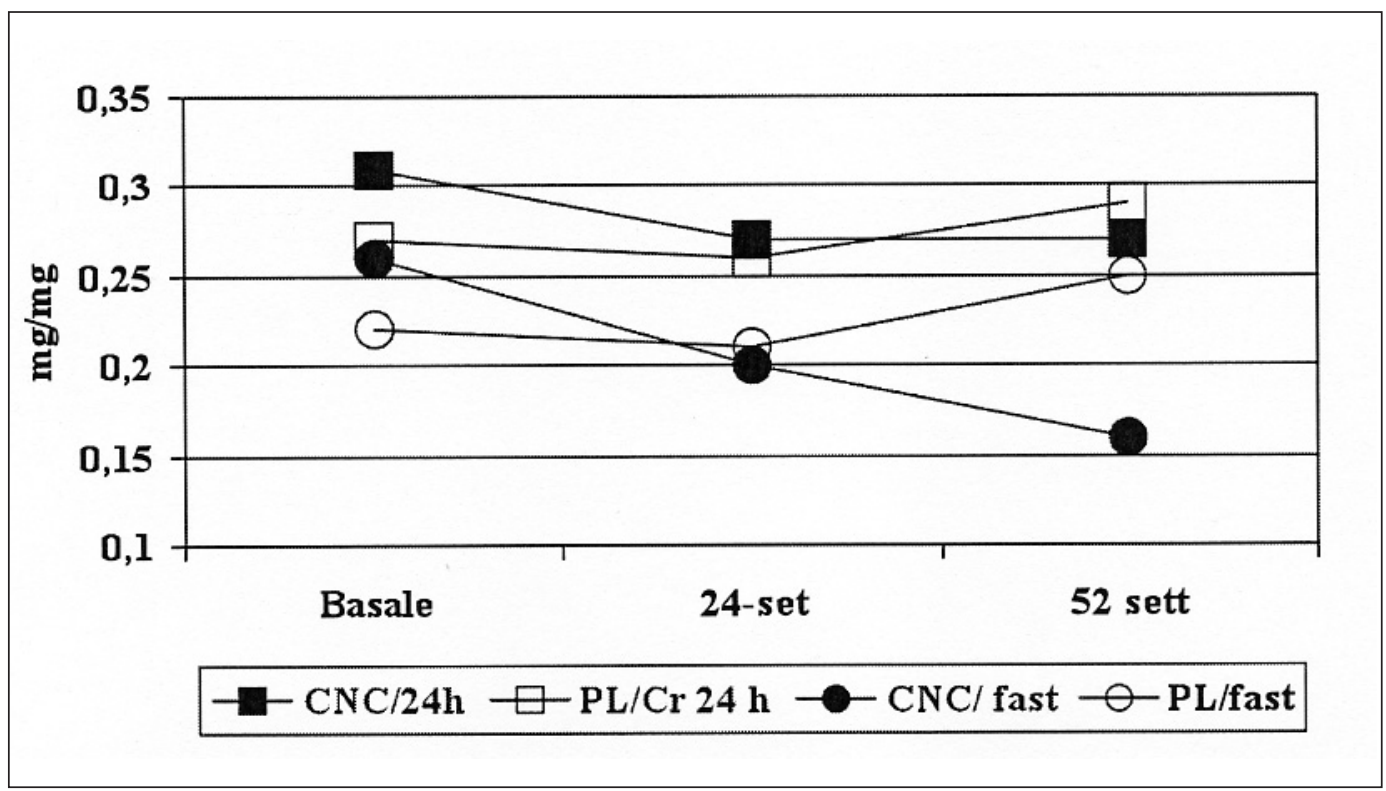

Fig. 2 - Andamento della calciuria su urine di $\mathbf{2 4}$ ore e su urine fasting in pazienti con IPTP trattati con Cinacalcet $(\mathrm{CN}$, nr. 40) o con placebo (PL, nr, 38).

l'aumento del PTH (8) e pertanto consiglia terapia con vitamina $D$.

SERM. I modulatori selettivi dei recettori degli estrogeni si sono dimostrati utili nella gestione di pazienti con IPTP ed osteoporosi post-menopausale. Il raloxifene, in due distinti studi, ha indotto una riduzione della calcemia e dei marker di riassorbimento osseo e un miglioramento del quadro densitometrico. Nessun effetto è stato notato sui livelli di PTH $(9,10)$.

Difosfonati. Attraverso una riduzione del turn over scheletrico, questi farmaci sono in grado di ridurre calcemia e calciuria, non solo nei pazienti con osteoporosi ipercalciurica ma anche in quelli con IPTP. L'alendronato, alla dose di $10 \mathrm{mg} / \mathrm{die}$, ha indotto un aumento densitometrico lombare e femorale $(11,12)$. Il transitorio aumento del PTH sierico tende a regredire nel lungo termine. Scarsi o nulli gli effetti su calcemia totale e ionizzata e sulla calciuria negli studi citati.

\section{Calcolosi Renale e IPTP}

Abbiamo ricordato che la nefrolitiasi è forse la complicanza più frequente in corso di IPTP. La patogenesi della nefrolitiasi ed i motivi per cui alcuni pazienti con IPTP facciano calcoli renali ed altri no, non sono del tutto chiari. Un lavoro recente ha trovato che la calcolosi renale era più frequente in pazienti con IPTP portatori di una variante allelica del CaSR associata a più severa ipercalciuria e minori livelli di PTH (13). Nella nostra esperienza clinica i pazienti con IPTP e calcoli sono quelli con funzione renale in genere nor- male, con elevazione significativa dei livelli di calcitriolo, conseguente ipercalciuria (e iperossaluria), relativo minore incremento di citraturia. Il combinarsi di questi fattori aumenta stabilmente la saturazione urinaria dei sali di calcio e facilita la calcolosi renale (Fig. 1). Quanto sopra spiega come più della metà dei pazienti con IPTP e calcolosi producano calcoli di calcio ossalato, un'altra quota calcoli misti, ed altri infine, calcoli di calcio fosfato.

Come detto la calcolosi renale è da considerarsi una delle complicazioni di IPTP, in cui vi è l'indicazione a PTX. Tuttavia situazioni, come quelle sopra elencate, in cui si attua un management medico, sono relativamente frequenti e impongono una corretta gestione della nefrolitiasi. A questo punto è da valutare la problematica del possibile beneficio del calciomimetico, che si potrebbe aggiungere alle raccomandazioni di trattamento medico già esposte.

\section{Calciomimetici nella terapia medica di IPTP}

Come noto i calciomimetici costituiscono una nuova classe di farmaci che agiscono come attivatori del calcium sensing receptor (CaSR), sia a livello paratiroideo che a livello renale. A parità di calcemia $\mathrm{i}$ calciomimetici inducono una soppressione della secrezione paratiroidea, con uno shift a sinistra della curva di set-point calcio/PTH.

Il Cinacalcet è oggi il calciomimetico più usato, specie nella terapia di HPT secondario (14). Recentemente è 
stato aggiunto in scheda tecnica il suo utilizzo anche nei pazienti con IPTP. Nei pochi lavori fin qui disponibili, a dosi di 30-60 mg/die si è ottenuta una riduzione dei livelli di calcemia e di PTH di circa il 50\% (15). Tuttavia, poiché il CaSR è espresso anche a livello tubulare renale, ove riduce il riassorbimento di calcio, una sua attivazione potrebbe peggiorare l'ipercalciuria. In altre parole, potrebbe realizzarsi una situazione analoga a quella delle ipercalciurie ipocalcemiche causate da mutazioni attivanti del CaSR (16).

In uno studio sperimentale su ratti geneticamente ipercalciurici Cinacalcet ridusse marcatamente PTH e calcemia e non indusse significative variazioni di calciuria nei ratti mantenuti a dieta normocalcica (17). In effetti l'escrezione frazionale di calcio risulta dall'interazione fra carico filtrato e riassorbimento tubulare. Il Cinacalcet agisce su entrambi i parametri, riducendo il primo, attraverso una riduzione della calcemia, ed il secondo con la sua azione a livello tubulare distale. Un altro possibile interferente è lo stato del riassorbimento scheletrico che, con la riduzione dei livelli di PTH, potrebbe ridursi in modo significativo e contribuire alla riduzione della calcemia e di qui del carico filtrato di calcio. Infine, tenuto conto che il PTH di per sé attiva la 1-alfa-drossilasi renale e quindi il calcitriolo sierico, ma aumenta anche il riassorbimento tubulare distale, una sua riduzione, indotta da Cinacalcet, potrà avere effetti opposti, di entità variabile, sul bilancio esterno del calcio. Il risultato netto sulla calciuria dipenderà dal bilancio delle molteplici e contrapposte interazioni suddette.

I risultati ottenuti in uno studio prospettico di Peacock et al (18), riportavano scarse variazioni della calciuria nei pazienti trattati con 30-60 mg/die di Cinacalcet. Tuttavia, la calciuria fasting si riduceva in modo significativo $(p<0.001)$ nei pazienti trattati (Fig. 2). In quella casistica la riduzione dei livelli di PTH non raggiungeva la significatività, al pari dei livelli di calcitriolo, mentre molto significativa era la riduzione della calcemia da $10.7 \pm 0.5$ a $9.7 \pm 0.5 \mathrm{mg} / \mathrm{dL}(\mathrm{p}<0.001)$. Nonostante un importante effetto di riduzione della calciuria fasting, non vi era alcuna variazione significativa dei parametri di metabolismo scheletrico. A tale proposito è stato di recente ribadito che i calciomimetici, in pazienti con IPTP, non hanno effetti significativi sia sul turnover scheletrico che sulla densità minerale ossea (19). In relazione al rischio di calcolosi renale, che nei pazienti con IPTP, è causato dall'aumento dello stato di saturazione delle urine rispetto al calcio ossalato ( $\mathrm{Ca}$ $\mathrm{Ox}$ ) ed al calcio fosfato o brushite (ßbsh), non disponiamo ad oggi di alcun dato di letteratura. Nel già citato studio sperimentale sui ratti ipercalciurici il Ci- nacalcet non variava in modo significativo $\beta \mathrm{CaOx}$ e ßbsh (17). Tuttavia, i ratti geneticamente ipercalciurici non sono iperparatiroidei e pertanto quelle conclusioni non possono essere traslate su IPTP nell'uomo.

\section{Conclusioni}

I dati disponibili non consentono pertanto conclusioni sui vantaggi dell'uso di calciomimetici nella nefrolitiasi in corso di IPTP. Sarebbe pertanto auspicabile progettare uno studio prospettico che arruoli pazienti con IPTP in cui, nella valutazione degli effetti di Cinacalcet, si tenga conto non solo dei parametri di metabolismo minerale, ma anche di tutti quelli rilevanti per il rischio litogeno complessivo. Questi dovranno includere tutti i parametri che concorrono allo stato di saturazione, inclusi quindi, oltre a calciuria ed ossaluria, anche $\mathrm{pH}$, volume urinario e citraturia.

Ad oggi possiamo affermare con sufficiente certezza che la paratiroidectomia associa ad una significativa riduzione del rischio di calcolosi calcica, anche una frequente remissione o riduzione delle recidive $(7,20)$, se questo sia vero anche per la terapia con calciomimetici non è ad oggi affermabile.

Indirizzo degli Autori:

Martino Marangella, MD

S.C. Nefrologia e Dialisi

A.O. Ordine Mauriziano Umberto I

Largo Turati, 62

10128 Torino

mmarangella@mauriziano.it 


\section{Bibliografia}

1. Silverberg SJ, Bilezikian JP. Incipient primary hyperparathyroidism: a "forme fruste" of an old disease. J Clin Endocrinol Metab 2003; 88: 5348-52.

2. Silverberg SJ, Shane E, Jacobs TP, Siris ES, Bilezikian JP. A ten-year prospective study of primary hyperparathyroidism with or without parathyroid surgery. $\mathrm{N}$ Engl J Med 1999; 341: 1249-55.

3. Rubin MR, Bilezikian JP, McMahon DJ, et al. The natural history of primary hyperparathyroidism with or without parathyroid surgery after 15 years. J Clin Endocrinol Metab 2008; 93: 3462-70.

4. Ambrogini E, Cetani F, Cianferotti L, et al. Surgery or surveillance for mild asymptomatic primary hyperparathyroidism: a prospective, randomized clinical trial. J Clin Endocrinol Metab 2007; 92: 3114-21.

5. Bilezikian JP, Potts JT, Fuleihan GEH, et al. Summary statement from a workshop on asymptomatic primary hyperparathyroidism: a perspective for the 21st century. J Clin Endocrinol Metab 2002; 87: 5353-61.

6. Bilezikian JP, Khan AA, Potts JT Jr; Third International Workshop on the Management of Asymptomatic Primary Hyperthyroidism. Guidelines for the management of asymptomatic primary hyperparathyroidism: summary statement from the third international workshop. J Clin Endocrinol Metab 2009; 94: 335-9.

7. Mollerup CL, Vestergaard P, Frøkjaer VG, Mosekilde L, Christiansen P, Blichert-Toft M. Risk of renal stone events in primary hyperparathyroidism before and after parathyroid surgery: controlled retrospective follow up study. BMJ 2002; 325: 807.

8. Souberbielle JC, Lawson-Body E, Hammadi B, Sarfati $\mathrm{E}$, Kahan A, Cormier C. The use in clinical practice of parathyroid hormone normative values established in vitamin D-sufficient subjects. J Clin Endocrinol Metab 2003; 88: 3501-4.

9. Rubin MR, Lee KH, McMahon DJ, Silverberg SJ. Raloxifene lowers serum calcium and markers of bone turnover in postmenopausal women with primary hyperparathyroidism. J Clin Endocrinol Metab 2003; 88: 1174-8.

10. Zanchetta JR, Bogado CE. Raloxifene reverses bone loss in postmenopausal women with mild asymptomatic primary hyperparathyroidism. J Bone Miner Res 2001; 16: 189-90.

11. Chow CC, Chan WB, Li JKY, et al. Oral alendronate increases bone mineral density in postmenopausal women with primary hyperparathyroidism. J Clin Endocrinol Metab 2003; 88: 581-7.

12. Khan AA, Bilezikiam JP, Kung AW, Ahmed MM, Dubois SJ, Ho AY, et al. Alendronate in primary hyperparathyroidism: a double-blind, randomized, placebo-controlled trial. J Clin Endocrinol Metab 2004; 89: 3319-25.

13. Scillitani A, Guarnieri V, Battista C, et al. Primary Hyperparathyroidism and the Presence of Kidney Stones Are Associated with Different Haplotypes of the Calcium-Sensing Receptor. J Clin Endocrinol Metab 2007; 92: 277-83.

14. Block GA, Martin KJ, de Francisco AL, et al. Cinacalcet for secondary hyperparathyroidism in patients receiving hemodialysis. N Engl J Med 2004; 350: 1516-25.

15. Shoback DM, Bilezikian JP, Turner SA, et al. The calcimimetic Cinacalcet normalizes serum calcium in subjects with primary hyperparathyroidism. J Clin Endocrinol Metab 2003; 88: 5644-9.

16. Stechman MJ, Loh NY, Thakker RV. Genetics of hypercalciuric nephrolithiasis: renal stone disease. Ann N Y Acad Sci 2007; 1116: 461-84.

17. Bushinsky DA, Laplante K, Asplin JR. Effect of cinacalcet on urine calcium excretion and supersaturation in genetic hypercalciuric stone-forming rats. Kidney Int 2006; 69: 1586-92.

18. Peacock M, Bilezikian JP, Klassen PS et al. Cinacalcet hydrochloride maintains long-term normocalcemia in patients with primary hyperparathyroidism. J Clin Endocrinol Metab 2005; 90: 135-41.

19. Khan A, Grey A, Shoback D. Medical management of asymptomatic primary hyperparathyroidism: proceedings of the third international workshop. J Clin Endocrinol Metab 2009; 94: 373-81.

20. Vitale C, Marangella M, Varvello G, et al. Effects of parathyroidectomy on citrate excretion in patients with primary hyperparathyroidism and calcium nephrolithiasis. Ital J Mineral Electrolyte Metab 1992; 6: 109-12. 